\title{
Au-delà de la grippe : Tendances des éclosions d'infections respiratoires dans les établissements de soins de santé de l'Ontario de 2007 à 2017, et implications pour la gestion des éclosions non grippales
}

\author{
Katherine Paphitis ${ }^{1}$, Camille Achonu ${ }^{1 *}$, Sandra Callery ${ }^{1}$, Jonathan Gubbay ${ }^{1,2}$, Kevin Katz ${ }^{3}$, \\ Matthew Muller ${ }^{4}$, Herveen Sachdeva ${ }^{5}$, Bryna Warshawsky ${ }^{1,6}$, Michael Whelan', Gary Garber ${ }^{1}, 7$, \\ Michelle Murti ${ }^{1,2}$
}

\section{Résumé}

Contexte : Les éclosions provoquent une morbidité et une mortalité importantes dans les établissements de soins de santé. Les méthodes de dépistage actuelles permettent de cerner des agents pathogènes respiratoires viraux spécifiques, mais l'approche de la gestion des éclosions reste générale.

Objectifs : Notre objectif était d'examiner les tendances spécifiques aux agents pathogènes dans les éclosions respiratoires, y compris la façon dont le taux d'attaque, le taux de létalité et la durée de l'éclosion diffèrent par agent pathogène entre les hôpitaux, et les établissements de soins de longue durée et les maisons de retraite en Ontario.

Méthodes : Les éclosions d'infections respiratoires confirmées dans les hôpitaux et les établissements de soins de longue durée ou les maisons de retraite de l'Ontario signalées entre le ${ }^{\text {1er }}$ septembre 2007 et le 31 août 2017 ont été extraites du Système intégré d'information sur la santé publique (SIISP). Les taux d'attaque médians, la durée des éclosions et les taux généraux de létalité des éclosions spécifiques à un pathogène ont été comparés dans les deux types d'établissements.

Résultats : Au cours de la période de surveillance de 10 ans, 9870 éclosions respiratoires confirmées ont été signalées dans les hôpitaux et les établissements de soins de longue durée ou les maisons de retraite de l'Ontario. La grippe était à l'origine de la plupart des éclosions (32\% dans les établissements de soins de longue durée et les maisons de retraite, 51 \% dans les hôpitaux), mais ces éclosions étaient plus courtes et présentaient des taux d'attaque inférieurs à ceux de la plupart des éclosions non grippales dans les deux types d'établissements. Le métapneumovirus humain, bien que peu fréquent (moins de $4 \%$ des éclosions), présentait des taux de létalité élevés dans les deux types d'établissements.

Conclusion : Les taux d'attaque et les taux de létalité variaient selon l'agent pathogène, tout comme la durée de l'éclosion. L'élaboration de conseils propres à la gestion des éclosions, qui tiennent compte de l'agent pathogène et de l'établissement de soins de santé, pourrait être utile pour limiter la charge des éclosions respiratoires.
Cette oeuvre est mise à la disposition selon les termes de la licence internationale Creative Commons Attribution 4.0

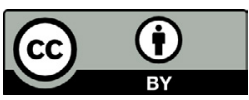

Affiliations

1 Santé publique Ontario, Toronto, ON

2 Université de Toronto, Toronto, $\mathrm{ON}$

${ }^{3}$ Hôpital général de North York, Toronto, ON

${ }^{4}$ Hôpital St Michael, Toronto, ON

${ }^{5}$ Santé publique de Toronto, Toronto, ON

${ }^{6}$ Université Western, London, ON

${ }^{7}$ Université d'Ottawa, Ottawa, ON

*Correspondance :

camille.achonu@oahpp.ca

Citation proposée : Paphitis K, Achonu C, Callery S, Gubbay J, Katz K, Muller M, Sachdeva H, Warshawsky B, Whelan M, Garber G, Murti M. Au-delà de la grippe : Tendances des éclosions d'infections respiratoires dans les établissements de soins de santé de l'Ontario de 2007 à 2017, et implications pour la gestion des éclosions non grippales. Relevé des maladies transmissibles au Canada 2021;47(5/6):294-301.

https://doi.org/10.14745/ccdr.v47i56a04f

Mots-clés : éclosions d'une maladie, établissements de soins de santé, établissement de soins de longue durée, métapneumovirus, grippe, humain, morbidité 


\section{Contexte}

Les éclosions d'infections respiratoires dues à des agents pathogènes viraux tels que la grippe, les coronavirus saisonniers et les rhinovirus sont fréquentes dans les établissements de soins de santé où les contacts étroits sont multiples et où le risque de complications est accru en raison de l'âge, d'une maladie sous-jacente ou d'autres facteurs $(1,2)$. En outre, les éclosions respiratoires font peser une lourde charge sur le système de soins de santé chaque saison, une éclosion confirmée dans un établissement de soins de santé ayant des répercussions sur les admissions et les transferts de patients ou de résidents, ainsi que sur les affectations du personnel.

En Ontario, les éclosions d'infections respiratoires dans les institutions [y compris les établissements de soins de longue durée et les maisons de retraite] et les hôpitaux publics doivent être signalées aux unités de santé publique locales pour qu'elles soient surveillées et reçoivent des conseils sur la gestion des éclosions (3-5). La plupart des directives relatives aux éclosions d'infections respiratoires (appelées " éclosions respiratoires ») en Ontario sont fondées sur des mesures générales de prévention et de contrôle des infections en utilisant la grippe comme modèle $(6,7)$.

Étant donné la disponibilité d'interventions spécifiques pour prévenir la transmission de la grippe pendant les éclosions (e.g. la prophylaxie antivirale), les tests de laboratoire pour les éclosions respiratoires en Ontario ont été principalement axés sur l'identification de la grippe. Avant la pandémie de grippe A [(H1N1) pdm09] de 2009, les principales méthodes de dépistage des agents pathogènes respiratoires étaient la culture virale et les tests de détection rapide de la grippe. Les écouvillons nasopharyngés prélevés sur les résidents des établissements de soins de longue durée et les maisons de retraite, et des établissements de soins de santé sont généralement soumis au laboratoire de santé publique provincial pour analyse, tandis que les hôpitaux peuvent soumettre les échantillons de l'éclosion au laboratoire de santé publique ou à leur propre laboratoire. L'évolution des capacités d'analyse du laboratoire provincial de santé publique, comme l'introduction de la transcription inverse en temps réel - réaction en chaîne par polymérase (RT-PCR) en 2009 et d'un panel viral respiratoire multiplex en 2010, a permis de dépister et d'identifier de manière plus sensible et plus rapide de multiples agents pathogènes respiratoires. Des changements similaires ont été mis en œuvre dans les laboratoires hospitaliers et communautaires de l'Ontario, $23 \%$ de ces laboratoires ayant déclaré effectuer des tests moléculaires multiplex en 2017 (8). Une meilleure capacité à différencier les agents pathogènes responsables permet d'adapter les mesures de prévention et de contrôle des infections à un agent pathogène spécifique et peut réduire la nécessité de mesures de contrôle inutiles ou trop restrictives dans certaines situations.
L'objectif de cette étude était d'examiner et de décrire les taux d'attaque, la durée des éclosions et les taux de létalité associés aux agents pathogènes des éclosions respiratoires, et de voir comment ils diffèrent entre les hôpitaux, et les établissements de soins de longue durée et les maisons de retraite.

\section{Méthodes}

En Ontario, les éclosions respiratoires signalées par les établissements aux unités locales de santé publique sont saisies dans le Système intégré d'information sur la santé publique (SIISP). Dans cette étude, nous n'avons analysé que les éclosions répondant à la définition provinciale d'une éclosion respiratoire confirmée, c'est-à-dire deux cas d'infection respiratoire aiguë dans les 48 heures et ayant un lien épidémiologique commun, dont au moins un est confirmé en laboratoire; ou trois cas d'infection respiratoire aiguë dans les 48 heures, ayant un lien épidémiologique commun et sans confirmation en laboratoire (9). Les éclosions survenues dans des établissements de soins de longue durée ou les maisons de retraite ont été combinées en une seule catégorie (SDL). Les informations rapportées qui ont été utilisées dans nos analyses comprenaient les identifiants des établissements, les dates d'apparition de la maladie chez le premier et le dernier cas identifiés, les dates de déclaration de l'éclosion, les résultats de laboratoire, le nombre total de cas de patients ou résidents, le nombre total de patients ou résidents à risque et les décès parmi les cas d'éclosion $(3,4)$.

Les éclosions confirmées signalées dans les établissements entre le $1^{\text {er }}$ septembre 2007 et le 31 août 2017 ont été extraites du SIISP. Les saisons d'éclosions respiratoires ont été définies comme allant du $1^{\text {er }}$ septembre au 31 août de l'année suivante, en fonction de la date de déclaration de l'éclosion ou de la date de saisie de l'éclosion dans le système SIISP si la date de déclaration était manquante $(n=67)$. La durée de l'éclosion a été définie comme la période en jours entre la date d'apparition des symptômes pour le premier cas identifié et celle du dernier cas identifié. Les éclosions pour lesquelles la date d'apparition du premier ou du dernier cas signalé était manquante ou improbable ont été exclues des analyses portant sur la durée $(n=704)$.

Les taux d'attaque ont été calculés comme le nombre de cas de patients ou résidents divisé par le nombre total de patients ou résidents à risque dans la zone affectée (e.g. l'ensemble de l'établissement, de l'étage ou de l'unité). Les taux de létalité ont été calculés comme étant le nombre de décès liés à l'éclosion divisé par le nombre total de cas de résidents ou patients, et multiplié par 100. Les foyers présentant des valeurs improbables, comme des taux d'attaque ou des taux de létalité supérieurs à $100 \%$, ont été exclus des analyses des taux d'attaque et des taux de létalité $(n=341)$. 
Les éclosions dues aux entérovirus, aux rhinovirus ou aux entérovirus et rhinovirus ont été regroupées en une seule catégorie, "entéro/rhinovirus ", et les virus de la grippe ( $A, B$ ou $A$ et $B$ ) ont été regroupés en une seule catégorie, "grippe ». Les éclosions où plus d'un agent pathogène a été détecté ont été classées comme " multiples ». Les éclosions dues à l'adénovirus ont été exclues de certaines analyses en raison de leur faible nombre $(n=5)$. Les éclosions pour lesquels aucun agent pathogène spécifique n'a été identifié ont été classées dans la catégorie « inconnue ».

Les analyses ont été effectuées au SAS 9.4 (SAS Institute Inc., Cary, Caroline du Nord, États-Unis). En ce qui concerne I'importance statistique, les différences de durée médiane des éclosions et de taux médian d'attaque des résidents ou patients entre les hôpitaux et les établissements de soins de longue durée/maisons de retraite pour chaque agent pathogène ont été évaluées à l'aide du test de Wilcoxon-Mann-Whitney pour obtenir une différence entre les médianes. Dans l'ensemble, les taux de létalité spécifiques à un agent pathogène dans l'un ou I'autre type d'établissement ont été comparés à l'aide du test du chi carré de Pearson ou du test exact de Fisher. Pour toutes les analyses, le niveau d'importance statistique était de $5 \%$ $(\alpha=0,05)$.

L'approbation par le comité de l'éthique de la recherche n'a pas été requise pour ce projet, car les activités décrites ici sont considérées comme une surveillance de routine (10).

\section{Résultats}

En date du 31 décembre 2019, l'Ontario comptait 151 hôpitaux et 1392 établissements de soins de longue durée et maisons de retraite opérationnels (11). Au cours des 10 années de surveillance, 9870 éclosions de maladies respiratoires sont survenues dans les hôpitaux, et les établissements de soins de longue durée et maisons de retraite de l'Ontario (tableau 1). La plupart des éclosions ont eu lieu dans des établissements de soins de longue durée ou des maisons de retraite $(92,0 \%)$; 1210 établissements de soins de longue durée ou maisons de retraite ont signalé une ou plusieurs éclosions respiratoires pendant la période d'étude $(86,9 \%)$. Le nombre d'éclosions signalées par les différents établissements de soins de longue durée ou maisons de retraite varie de 1 à 139, avec une médiane de cinq éclosions par établissement. En revanche, 93 hôpitaux ont signalé une ou plusieurs éclosions respiratoires pendant la période d'étude (61,6\%). Le nombre d'éclosions signalées par un seul hôpital allait de 1 à 133 sur la période d'étude de 10 ans, avec une médiane de deux éclosions par hôpital.

Le virus de la grippe était l'agent pathogène le plus fréquemment signalé dans les deux types d'établissements (31,9\% dans les établissements de soins de longue durée et les
Tableau 1 : Éclosions respiratoires confirmées dans les établissements institutionnels de soins de santé, par agent pathogène, Ontario, 2007-2008 à 2016-2017 $(n=9$ 870)

\begin{tabular}{|c|c|c|c|c|c|c|}
\hline \multirow{3}{*}{$\begin{array}{c}\text { Agent } \\
\text { pathogène }\end{array}$} & \multicolumn{6}{|c|}{$\begin{array}{c}\text { Nombre et proportion d'éclosions dans les } \\
\text { établissements institutionnels de soins de } \\
\text { santé }\end{array}$} \\
\hline & \multicolumn{2}{|c|}{ Hôpitaux } & \multicolumn{2}{|c|}{$\begin{array}{l}\text { Établissements } \\
\text { de SDL/ } \\
\text { maisons de } \\
\text { retraite }\end{array}$} & \multicolumn{2}{|c|}{ Total } \\
\hline & $\mathbf{n}$ & $\%$ & $\mathbf{n}$ & $\%$ & $\mathbf{n}$ & $\%$ \\
\hline Adénovirus & 1 & 0,1 & 4 & 0,0 & 5 & 0,1 \\
\hline Coronavirus & 17 & 2,2 & 499 & 5,5 & 516 & 5,2 \\
\hline Entéro/rhinovirus & 87 & 11,1 & 1654 & 18,2 & 1741 & 17,6 \\
\hline Grippe & 401 & 51,0 & 2900 & 31,9 & 3301 & 33,4 \\
\hline MPVh & 25 & 3,2 & 357 & 3,9 & 382 & 3,9 \\
\hline $\begin{array}{l}\text { Virus } \\
\text { parainfluenza }\end{array}$ & 54 & 6,9 & 482 & 5,3 & 536 & 5,4 \\
\hline VRS & 70 & 8,9 & 560 & 6,2 & 630 & 6,4 \\
\hline $\begin{array}{l}\text { Agents } \\
\text { pathogènes } \\
\text { multiples }\end{array}$ & 77 & 9,8 & 1085 & 11,9 & 1162 & 11,8 \\
\hline $\begin{array}{l}\text { Agents } \\
\text { pathogènes } \\
\text { inconnus }\end{array}$ & 54 & 6,9 & 1543 & 17,0 & 1597 & 16,2 \\
\hline Total & 786 & 100,0 & 9084 & 100,0 & 9870 & 100,0 \\
\hline
\end{tabular}

maisons de retraite, et 51,0 \% dans les hôpitaux). Les éclosions dues au coronavirus, à l'entéro/rhinovirus et à des agents pathogènes multiples ont augmenté au cours de la période d'étude, tandis que l'incidence des éclosions dues à un agent pathogène inconnu a diminué de 2008-2009 à 2009-2010 et s'est stabilisée ces dernières années (figure 1). Dans les hôpitaux, et les établissements de soins de longue durée et les, après 2009-2010, le nombre d'éclosions de grippe a affiché un profil cyclique, avec un nombre plus élevé d'éclosions de grippe une année sur deux, par rapport aux années précédentes et suivantes (figure 1 ).

\section{Taux d'attaque}

Les taux d'attaque médians pour chaque agent pathogène étaient généralement plus élevés dans les hôpitaux que dans les établissements de soins de longue durée et les maisons de retraite et étaient les plus élevés dans les hôpitaux pour les éclosions dues aux coronavirus $(22,5 \%)$ et au virus parainfluenza $(22,0 \%)$, et les plus élevés dans les établissements de soins de longue durée et les maisons de retraite pour les épidémies dues au métapneumovirus humain (MPVh) (18,0 \%) (tableau 2). La différence de taux d'attaque médian entre les établissements de soins de longue durée et les maisons de retraite, et les 
Figure 1 : Éclosions respiratoires confirmées dans les établissements institutionnels de soins de santé, par agent pathogène et par saison, Ontario, 2007-2008 à 2016-2017 ( $n=9$ 870)

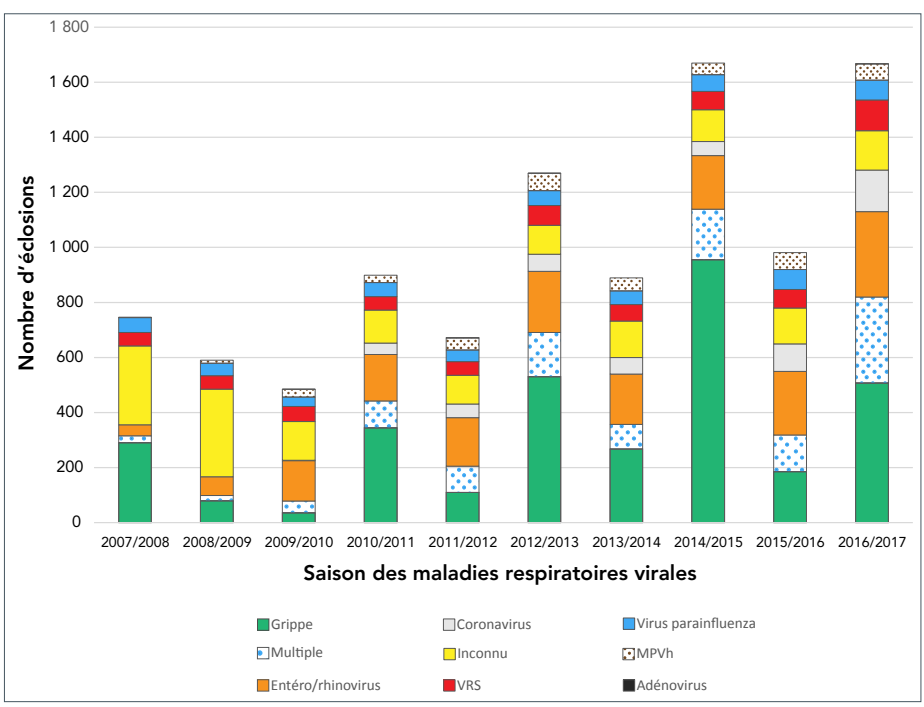

Abréviations : MPVh, métapneumovirus humain; VRS, virus respiratoire syncytial

hôpitaux était bien plus importante pour les éclosions dues à l'entéro/rhinovirus, au MPVh, au virus parainfluenza, à des pathogènes multiples et lorsque le pathogène causal était inconnu (tableau 2).

\section{Durée de l'éclosion}

La durée de l'éclosion variait de 0 à 105 jours dans les hôpitaux et de 0 à 122 jours dans les établissements de soins de longue durée et les maisons de retraite. La différence de durée médiane des éclosions entre les établissements de soins de longue durée et les maisons de retraite, et les hôpitaux n'était significativement différente que pour les éclosions dues à la grippe $(8,0$ jours dans les établissements de soins de longue durée et les maisons de retraite par rapport à 5,0 jours dans les hôpitaux; $P<0,001$ ) (tableau 2).

\section{Taux de létalité}

La plupart des éclosions n'ont entrainé aucun décès, et les taux de létalité globaux par agent pathogène dans l'un ou l'autre type d'établissement étaient relativement faibles (tableau 3). La grippe présentait le taux de létalité le plus élevé dans les établissements de soins de longue durée et les maisons de retraite, tandis que le virus de l'hépatite $C$ présentait le taux de létalité le plus élevé dans les hôpitaux (tableau 3). Les taux de létalité les plus faibles ont été observés dans les épidémies d'entéro/rhinovirus et de coronavirus dans les hôpitaux et les établissements de soins de longue durée et les maisons de retraite, respectivement (tableau 3). On a constaté une différence significative $(P=0,003)$ dans les taux de létalité globale des cas de grippe entre les hôpitaux $(2,35 \%)$ et les établissements de soins de longue durée et les maisons de retraite (3,54 \%), ainsi que pour les cas dus à des agents pathogènes inconnus (tableau 3).

Tableau 2 : Taux d'attaque médians et durée de l'épidémie pour les éclosions respiratoires dans les établissements institutionnels de soins de santé, par agent pathogène, Ontario, 2007-2008 à 2016-2017a

\begin{tabular}{|c|c|c|c|c|c|c|c|c|c|c|}
\hline \multirow[b]{2}{*}{$\begin{array}{c}\text { Agent } \\
\text { pathogène }\end{array}$} & \multicolumn{5}{|c|}{ Taux d'attaque médian (\%) } & \multicolumn{5}{|c|}{ Durée médiane de l'éclosion (jours) } \\
\hline & Hôpital & $\mathrm{EIQ}^{\mathrm{b}}$ & $\begin{array}{l}\text { Établissements } \\
\text { de soins de } \\
\text { longue durée et } \\
\text { les maisons de } \\
\text { retraite }\end{array}$ & $\mathrm{EIQ}^{\mathrm{b}}$ & valeur-p & Hôpital & $\mathrm{EIQ}^{\mathrm{b}}$ & $\begin{array}{l}\text { Établissements } \\
\text { de soins de } \\
\text { longue durée et } \\
\text { les maisons de } \\
\text { retraite }\end{array}$ & $\mathrm{EIQ}^{\mathrm{b}}$ & valeur-p \\
\hline Coronavirus & 22,5 & $10,5-33,5$ & 16,0 & $10,0-23,0$ & 0,284 & 7,0 & $4,0-12,0$ & 9,0 & $5,0-14,0$ & 0,283 \\
\hline Entéro/rhinovirus & 21,0 & $14,0-34,0$ & 16,0 & $10,0-24,0$ & $<0,001^{c}$ & 8,0 & $4,0-12,0$ & 8,0 & $5,0-13,0$ & 0,394 \\
\hline Grippe & 16,0 & $10,0-24,0$ & 15,0 & $9,0-24,0$ & 0,287 & 5,0 & $3,0-8,0$ & 8,0 & $5,0-12,0$ & $<0,001^{c}$ \\
\hline MPVh & 21,0 & $15,0-38,0$ & 18,0 & $11,0-27,0$ & $0,011^{c}$ & 10,0 & $5,0-14,0$ & 11,0 & $6,0-17,0$ & 0,163 \\
\hline Multiple & 20,5 & $14,0-33,0$ & 16,0 & $10,0-25,5$ & $0,001^{c}$ & 13,0 & $6,0-20,0$ & 12,0 & $7,0-19,0$ & 0,879 \\
\hline $\begin{array}{l}\text { Virus } \\
\text { parainfluenza }\end{array}$ & 22,0 & $15,0-37,0$ & 17,0 & $11,0-25,0$ & $0,001^{c}$ & 9,5 & $4,5-16,0$ & 10,0 & $5,0-16,0$ & 0,516 \\
\hline VRS & 18,0 & $11,0-28,0$ & 17,0 & $11,0-27,0$ & 0,461 & 10,0 & $5,0-16,0$ & 11,5 & $6,0-18,0$ & 0,081 \\
\hline $\begin{array}{l}\text { Agents } \\
\text { pathogènes } \\
\text { inconnus }\end{array}$ & 21,0 & $13,0-32,0$ & 15,0 & $9,0-23,0$ & $0,001^{c}$ & 7,0 & $3,0-13,0$ & 8,0 & $4,0-13,0$ & 0,405 \\
\hline
\end{tabular}

Abréviations : EIQ, intervalle interquartile; MPVh, métapneumovirus humain; SDL, soins de longue durée; VRS, virus respiratoire syncytial

a Les éclosions dues à l'adénovirus ont été exclues en raison du faible nombre de cas (moins de 5 éclosions dans l'un ou l'autre type d'établissement)

${ }^{\mathrm{b}}$ Intervalle interquartile du $25^{\mathrm{e}}$ au $75^{\mathrm{e}}$ percentile pour chaque valeur médiane

c Significatif à $P<0,05$ 
Tableau 3 : Comparaison des taux de létalité des cas pour les éclosions respiratoires dans les établissements institutionnels de soins de santé, par agent pathogène, Ontario, 2007-2008 à 2016-2017 (n = 9 844)

\begin{tabular}{|c|c|c|c|c|c|}
\hline \multirow{2}{*}{ Pathogènea } & \multicolumn{2}{|c|}{ Hôpital } & \multicolumn{2}{|c|}{$\begin{array}{l}\text { Établissements de } \\
\text { soins de longue } \\
\text { durée et les maisons } \\
\text { de retraite }\end{array}$} & \multirow{2}{*}{ valeur-p } \\
\hline & $\begin{array}{l}\text { Taux de } \\
\text { létalité } \\
(\%)\end{array}$ & $\begin{array}{l}\text { Nombre } \\
\text { de cas } \\
\text { signalés } \\
\text { (n) }\end{array}$ & $\begin{array}{l}\text { Taux de } \\
\text { létalité } \\
(\%)\end{array}$ & $\begin{array}{l}\text { Nombre } \\
\text { de cas } \\
\text { signalés } \\
\text { (n) }\end{array}$ & \\
\hline Coronavirus & 0,88 & 113 & 0,96 & 5815 & 1,000 \\
\hline $\begin{array}{l}\text { Entéro/ } \\
\text { rhinovirus }\end{array}$ & 0,82 & 612 & 1,62 & 20069 & 0,117 \\
\hline Grippe & 2,35 & 2296 & 3,54 & 41125 & $0,003^{b}$ \\
\hline MPVh & 3,43 & 175 & 3,25 & 4649 & 0,895 \\
\hline $\begin{array}{l}\text { Virus } \\
\text { parainfluenza }\end{array}$ & 2,78 & 431 & 2,12 & 6047 & 0,357 \\
\hline VRS & 2,65 & 415 & 2,39 & 7628 & 0,732 \\
\hline $\begin{array}{l}\text { Agents } \\
\text { pathogènes } \\
\text { multiples }\end{array}$ & 2,32 & 732 & 3,19 & 17912 & 0,189 \\
\hline $\begin{array}{l}\text { Agents } \\
\text { pathogènes } \\
\text { inconnus }\end{array}$ & 3,39 & 501 & 1,76 & 16040 & $0,007^{b}$ \\
\hline Total & 2,33 & 5275 & 2,64 & 119285 & s.o. \\
\hline
\end{tabular}

Abréviations : MPVh, métapneumovirus humain; SDL, soins de longue durée; s.o., sans objet; VRS, virus respiratoire syncytial

a Les éclosions dues à l'adénovirus ont été exclues en raison du faible nombre de cas (moins de 5 éclosions dans l'un ou l'autre type d'établissement)

b Significatif à $P<0,05$

\section{Discussion}

Chaque année, les éclosions d'infections respiratoires peuvent entraîner une morbidité et des perturbations importantes pour les résidents et les patients des établissements de soins de santé. Cette étude a révélé que $61,6 \%$ des hôpitaux et $86,9 \%$ des établissements de soins de longue durée et des maisons de retraite ont connu une ou plusieurs éclosions au cours de la période de surveillance, les établissements de soins de longue durée et les maisons de retraite ayant généralement un nombre plus élevé d'éclosions que les hôpitaux. Par rapport aux résidents des établissements de soins de longue durée et des maisons de retraite, les personnes hospitalisées présentant des symptômes respiratoires sont plus facilement isolées des autres dans une chambre individuelle, placées dans une cohorte avec d'autres personnes souffrant d'une maladie similaire ou renvoyées de l'établissement pour limiter leur potentiel de transmission au sein de ce dernier. À l'inverse, la mobilité indépendante accrue des résidents des établissements de soins de longue durée et des maisons de retraite, ainsi que la participation à des repas collectifs et à d'autres activités contribuent probablement à la transmission de la maladie dans ces établissements.

Les différences dans le nombre d'éclosions entre les types d'établissements peuvent également être dues à des différences dans la déclaration et la taille des établissements. L'évolution des tests d'échantillons au fil du temps a contribué à améliorer la détection des éclosions qui ne sont pas associées à la grippe. La capacité d'identifier et de différencier les agents pathogènes respiratoires non grippaux peut être utile pour la gestion et le contrôle des éclosions, en particulier compte tenu du degré variable de responsabilité des agents pathogènes individuels dans les éclosions et de la variabilité associée de la durée des éclosions, du taux d'attaque et du taux de létalité, comme observé dans cette étude.

En raison de la nature de la conception de l'établissement, les éclosions hospitalières peuvent être plus facilement limitées à de petites zones telles qu'un service, une unité ou un étage, alors qu'une éclosion dans un établissement de soins de longue durée ou une maison de retraite est plus susceptible de se produire dans l'ensemble de l'établissement, ce qui augmente le nombre de résidents et de membres du personnel exposés à la maladie et réduit le taux d'attaque global. Cela peut avoir contribué aux taux d'attaque plus élevés dans les hôpitaux par rapport aux établissements de soins de longue durée et aux maisons de retraite pour les épidémies dues à chaque pathogène respiratoire viral examiné dans cette étude.

En outre, les différences dans la proportion d'éclosions respiratoires dues à des agents pathogènes inconnus peuvent être attribuées aux différents algorithmes de test utilisés dans les hôpitaux, et les établissements de soins de longue durée et maisons de retraite, étant donné que ces derniers dépendent principalement du laboratoire de santé publique pour les tests, tandis que de nombreux hôpitaux effectuent leurs propres tests et peuvent avoir des critères de dépistage différents.

Si, dans cette étude, la plupart des éclosions respiratoires dans les établissements de soins de longue durée et les maisons de retraite, ainsi que dans les hôpitaux étaient dues à la grippe, les éclosions de grippe avaient également la durée médiane la plus courte de toutes les éclosions hospitalières et l'une des plus courtes pour les établissements de soins de longue durée et les maisons de retraite. La durée médiane des épidémies dues à la grippe était également significativement plus faible dans les hôpitaux que dans les établissements de soins de longue durée et les maisons de retraite. On sait que l'introduction précoce d'antiviraux, tant pour le traitement que pour la prophylaxie des éclosions de grippe, permet de les maîtriser rapidement, d'en réduire la durée et d'abaisser le taux d'attaque (12-14). Le respect des directives existantes en matière de gestion des éclosions de grippe, qui indiquent la fourniture d'antiviraux à tous les patients ou résidents, a probablement contribué aux faibles taux d'attaque et à la courte durée de l'éclosion observés dans cette étude.

Nous avons observé que les pics des éclosions de grippe suivaient une tendance biennale correspondant aux saisons dominantes de la grippe A (H3N2). Cette constatation est conforme à des recherches antérieures qui ont montré qu'il y a généralement un nombre accru d'éclosions de grippe en milieu hospitalier lors des années dominées par la grippe A (H3N2) (15). 
Selon les directives actuelles de l'Ontario en matière de santé respiratoire, la plupart des éclosions peuvent être déclarées terminées huit jours après l'apparition des symptômes chez le dernier patient ou résident identifié (ce qui correspond à une période d'incubation plus une période de transmission pour la grippe) (6). La grippe ayant la période d'incubation la plus courte des agents pathogènes examinés dans cette étude, la gestion de toutes les éclosions comme s'il s'agissait d'éclosions de grippe pourrait signifier que les mesures de contrôle des éclosions sont levées trop tôt. L'identification d'un agent pathogène spécifique permet de prendre en compte les périodes d'incubation et de transmission propres à chaque agent pathogène au moment de déterminer quand déclarer la fin d'une éclosion, ce qui garantit que les mesures de contrôle restent en place pendant une durée appropriée.

Dans cette étude, les éclosions dues à des agents pathogènes multiples ont été associées à des taux d'attaque significativement plus élevés dans les hôpitaux que dans les établissements de soins de longue durée et les maisons de retraite. Les éclosions dues à des agents pathogènes multiples étaient également associées à la plus longue durée médiane des éclosions dans les deux types d'établissements. Cela peut être dû à la circulation simultanée d'agents pathogènes ou au chevauchement d'éclosions causées par différents agents pathogènes, ce qui augmente à la fois le risque de maladie chez les patients ou résidents et la complexité de la gestion des éclosions. Lorsque les éclosions sont dues à plusieurs agents pathogènes circulant simultanément, il serait prudent de mettre en place des mesures de contrôle en fonction de l'agent pathogène dont la période d'incubation ou de transmission est la plus longue.

Dans l'ensemble, les taux de létalité étaient significativement plus élevés dans les établissements de soins de longue durée et les maisons de retraite que dans les hôpitaux pour les éclosions dues à la grippe et aux agents pathogènes inconnus. Les résidents des établissements de soins de longue durée et des maisons de retraite peuvent être exposés à un risque accru de décès associé à la grippe, malgré l'utilisation de médicaments antiviraux, en raison de leur âge plus avancé et de la présence d'autres comorbidités, qui augmentent le risque de conséquences graves de la grippe, notamment de pneumonie et de décès $(16,17)$, et en raison de directives avancées qui peuvent empêcher de recevoir des soins en milieu hospitalier. Une étude réalisée par luliano et al. (2018) a constaté que les taux de surmortalité associés à la grippe augmentaient avec l'âge au Canada, les personnes âgées de 75 ans et plus présentant les taux de surmortalité associés à la grippe les plus élevés par rapport aux cohortes d'âge plus jeune (17).

Les épidémies dues au MPVh ont été associées au taux de létalité le plus élevé dans les hôpitaux et au deuxième taux de létalité le plus élevé dans les établissements de soins de longue durée et les maisons de retraite. Plusieurs éclosions dues au MPVh ont également été décrites dans la littérature, où elles ont entraîné des taux d'attaque et des taux de létalité élevés dans les établissements de soins de longue durée aux États-Unis, ce qui souligne l'importance des tests pour identifier le MPVh comme agent pathogène responsable, $d$ 'autant plus que le caractère saisonnier et les symptômes associés au MPVh sont similaires à ceux $d$ 'autres agents pathogènes respiratoires $(18,19)$. Bien que les éclosions dues au MPVh et au virus parainfluenza ne représentent qu'une faible proportion des éclosions dues à des agents pathogènes connus dans l'un ou l'autre des types d'établissements (moins de $4 \%$ ), leurs taux d'attaque médians comparativement élevés et le taux global élevé de létalité du MPVh peuvent justifier des directives spécifiques de gestion des éclosions, telles qu'un isolement plus strict, le placement dans des cohortes et la restriction des mouvements, qui pourraient contribuer à réduire les taux d'attaque et la mortalité subséquente dans les milieux hospitaliers, et les établissements de soins de longue durée et les maisons de retraite. Inversement, bien que les entéro/rhinovirus aient été une cause fréquente d'éclosions dans les hôpitaux, et les établissements de soins de longue durée et les maisons de retraite, ces éclosions ont été associées à des taux d'attaque, des taux de létalité et une durée d'éclosions généralement plus faibles que les éclosions associées à d'autres agents pathogènes. Les orientations spécifiques de gestion des éclosions pour ces agents pathogènes pourraient donc être potentiellement plus permissives.

\section{Forces et faiblesses}

Cette étude présente plusieurs limites. Les données ne représentent que les épidémies signalées aux unités de santé publique et enregistrées dans le SIISP. La classification de la population à risque et les taux d'attaque qui en résultent, ainsi que les taux de létalité, sont fondés sur les informations rapportées par les établissements individuels aux unités de santé publique et peuvent être sujets à des variations dans la surveillance et les rapports entre les établissements et le type d'établissement, ce qui aurait pu avoir un impact sur les comparaisons entre les établissements. En outre, la mise en œuvre des mesures de contrôle des éclosions peut varier d'un établissement à l'autre en raison de différences contextuelles qui peuvent avoir un impact sur la variabilité des résultats observés dans cette étude. Les pratiques d'analyse en laboratoire dans les établissements hospitaliers qui effectuent leurs propres analyses d'échantillons peuvent varier d'une région à l'autre de la province, et au fil du temps, à mesure que les pratiques d'analyse évoluent. Étant donné que les algorithmes actuels de dépistage des éclosions sont hiérarchisés et initialement axés sur l'identification ou l'exclusion de la grippe, certaines éclosions dues à plusieurs agents pathogènes peuvent avoir été classées à tort comme des éclosions de grippe uniquement.

\section{Conclusion}

Étant donné le fardeau croissant des éclosions respiratoires sur le système de santé provincial en Ontario et ailleurs, cette étude met en évidence les possibilités où la détection rapide des éclosions, la confirmation des agents pathogènes et la mise en œuvre de mesures de contrôle des éclosions spécifiques aux 
agents pathogènes peuvent avoir des implications positives pour limiter la transmission de la maladie et la durée des éclosions. Les directives actuelles sur les éclosions en Ontario et aux États-Unis sont axées sur la grippe $(13,20)$.

En outre, des conseils spécifiques pour les hôpitaux, reconnaissant les différents défis dans les établissements de soins aigus et dans les établissements de soins de longue durée et les maisons de retraite, peuvent soutenir les pratiques de gestion des éclosions dans ces types d'établissements. Plusieurs provinces canadiennes ont élaboré des lignes directrices sur les éclosions de maladies respiratoires pour les établissements de soins aigus et les établissements de soins de longue durée et les maisons de retraite, y compris des recommandations pour la prise en charge des patients présentant des pathogènes spécifiques $(21,22)$, un modèle potentiel à prendre en compte dans l'élaboration de ressources applicables localement pour guider la gestion des éclosions de maladies respiratoires.

Bien que les données analysées pour cette étude aient précédé la collecte de données sur les éclosions dues au coronavirus du syndrome respiratoire aigu sévère 2 (SRAS-CoV-2), on prévoit que la mise en œuvre généralisée de mesures de prévention et de contrôle des infections visant à empêcher la transmission du SRAS-CoV-2 dans les établissements pendant la pandémie de 2020 aura eu un avantage supplémentaire en réduisant la transmission d'autres pathogènes respiratoires transmis par gouttelettes ou contact. Cet avantage devrait être le plus évident dans les établissements de soins de longue durée et les maisons de retraite, où les établissements ont probablement moins d'expertise sur place et de ressources humaines consacrées aux activités de prévention et de contrôle des infections.

Cette étude constitue un comparateur et une base de référence précieux pour les études futures visant à évaluer l'impact plus large des mesures de prévention et de contrôle du SRAS-CoV-2 dans les établissements.

\section{Déclaration des auteurs}

K. P. - A analysé et interprété les données de l'éclosion et a largement contribué à la rédaction du manuscrit

C. A. - A analysé et interprété les données de l'éclosion et a largement contribué à la rédaction du manuscrit

S. C. - A contribué de manière substantielle à la conception du travail et à la révision du manuscrit

J. G. - A contribué de manière substantielle à la conception du travail, à l'interprétation des méthodes d'essai en laboratoire et à la révision du manuscrit

K. K. - A contribué de manière substantielle à la conception du travail et à la révision du manuscrit

M. M. - A contribué de manière substantielle à la conception du travail et à la révision du manuscrit

H. S. - A contribué de manière substantielle à la conception du travail et à la révision du manuscrit

B. W. - A contribué de manière substantielle à l'interprétation des données de l'éclosion et à la révision du manuscrit
M. W. - A contribué de manière substantielle à la conception du travail et à la révision du manuscrit

G. G. - A contribué de manière substantielle à la conception du travail et à la révision du manuscrit

M. M. - A contribué de manière substantielle à la conception du travail, à l'interprétation des données d'éclosion et à la révision du manuscrit

Tous les auteurs ont lu et approuvé le manuscrit final.

Le contenu de l'article et les points de vue qui y sont exprimés n'engagent que les auteurs et ne correspondent pas nécessairement à ceux du gouvernement du Canada.

\section{Intérêts concurrents}

Les auteurs déclarent qu'ils n'ont pas d'intérêts concurrents.

\section{Financement}

Cette étude n'a bénéficié d'aucun financement externe. Tous les travaux de cette étude ont été réalisés à Santé publique Ontario et ont bénéficié de son soutien.

\section{Références}

1. Longtin J, Marchand-Austin A, Winter AL, Patel S, Eshaghi A, Jamieson F, Low DE, Gubbay JB. Rhinovirus outbreaks in long-term care facilities, Ontario, Canada. Emerg Infect Dis $2010 ; 16(9): 1463-5$. DOl PubMed

2. Childs A, Zullo AR, Joyce NR, McConeghy KW, van Aalst R, Moyo P, Bosco E, Mor V, Gravenstein S. The burden of respiratory infections among older adults in long-term care: a systematic review. BMC Geriatr 2019;19(1):210. DOl PubMed

3. Protection et la promotion de la santé (Loi sur la), L.R.O. 1990, c. H.7. Toronto (ON) : Gouvernement de l'Ontario (accédé 2019-10-25). https://www.ontario.ca/fr/lois/ loi/90h07

4. R.R.O. 1990, Règl. 569 : RAPPORTS. Toronto (ON) : Gouvernement de l'Ontario; 2018 (accédé 2019-10-25). https://www.ontario.ca/fr/lois/reglement/900569

5. Ministry of Health and Long-Term Care. Infectious diseases protocol. Appendix A: disease-specific chapters. Chapter: respiratory infection outbreaks in institutions and public hospitals. Toronto (ON): MoHLTC; 2019 (accédé 2019-10-25). http://www.health.gov.on.ca/en/pro/programs/publichealth/ oph_standards/docs/respiratory_outbreaks_chapter.pdf

6. Ministry of Health and Long-Term Care. Control of respiratory infection outbreaks in long-term care homes. Toronto (ON): MoHLTC; 2018 (accédé 2019-10-25). http://www.health.gov.on.ca/en/pro/programs/publichealth/ oph_standards/docs/reference/RESP_Infectn_ctrl_guide_ LTC_2018_en.pdf 
7. Ontario Hospital Association and the Ontario Medical Association Joint Communicable Diseases Surveillance Protocols Committee. Influenza surveillance protocol for Ontario hospitals. Toronto (ON): Ontario Hospital Association; 2018 (accédé 2019-10-25). https://www.oha. com/Documents/Influenza\%20Protocol\%20(November\%20 2018).pdf

8. Kozak R, Kerr E, Richardson D, Vermeiren C, Poutanen SM. How modern is your microbiology laboratory? Results of the 2017 Institute for Quality Management in Healthcare (IOHM) patterns of practice survey. (Presentation). 2019 (accédé 2019-10-25). https://iqmh.org/Portals/0/ Docs/Resources/Poster\%20-\%20MICRO\%20-\%20SUNCPHM-826\%20How\%20Modern\%20ls\%20Your\%20Lab\%20 Oral.pdf

9. Ministry of Health and Long-Term Care. Infectious diseases protocol. Appendix B: provincial case definitions for diseases of public health significance. Disease: respiratory infection outbreaks in institutions and public hospitals. Toronto (ON): MoHLTC; 2019b (accédé 2019-10-25). http://www.health. gov.on.ca/en/pro/programs/publichealth/oph_standards/ docs/respiratory_outbreaks_cd.pdf

10. Agence ontarienne de protection et de promotion de la santé (Loi de 2007 sur l'), L.O. 2007, chap. 10, annexe K. Toronto (ON) : Gouvernement de l'Ontario (accédé 2019-10-25). https://www.ontario.ca/fr/lois/ loi/07o10

11. Ontario Ministry of Health. Ministry of Long-Term Care. Ministry reports: master numbering system. Toronto (ON): MoHLTC; 2020 (accédé 2019-10-25). http://www.health.gov. on.ca/en/common/ministry/publications/reports/master_ numsys/master_numsys.aspx

12. Ye M, Jacobs A, Khan MN, Jaipaul J, Oda J, Johnson M, Doroshenko A. Evaluation of the use of oseltamivir prophylaxis in the control of influenza outbreaks in long-term care facilities in Alberta, Canada: a retrospective provincial database analysis. BMJ Open 2016;6(7):e011686. DOI PubMed

13. Centers for Disease Control and Prevention. Prevention strategies for seasonal influenza in healthcare settings: guidelines and recommendations. Atlanta (GA): CDC: 2018 (accédé 2019-10-25). https://www.cdc.gov/flu/professionals/ infectioncontrol/healthcaresettings.htm

14. Murti M, Fung CK, Chan K, Bigham M. Duration of influenza outbreaks in long-term care facilities after antiviral prophylaxis initiation: fraser Health, British Columbia, 2014-2017. Am J Infect Control 2018;46(9):1077-9. DOI PubMed

15. Murti M, Whelan M, Friedman L, Savic J, Johnstone J, Sider D, Warshawsky B. Éclosions de grippe dans les hôpitaux ontariens, 2012 à 2016. Relevé des maladies transmissibles au Canada 2018;44(9):277-32. DOI
16. Clay CC, Donart N, Fomukong N, Knight JB, Overheim K, Tipper J, Van Westrienen J, Hahn F, Harrod KS. Severe acute respiratory syndrome-coronavirus infection in aged nonhuman primates is associated with modulated pulmonary and systemic immune responses. Immun Ageing 2014;11(1):4. DOI PubMed

17. Iuliano AD, Roguski KM, Chang HH, Muscatello DJ, Palekar R, Tempia S, Cohen C, Gran JM, Schanzer D, Cowling BJ, Wu P, Kyncl J, Ang LW, Park M, Redlberger-Fritz M, Yu H, Espenhain L, Krishnan A, Emukule G, van Asten L, Pereira da Silva S,

Aungkulanon S, Buchholz U, Widdowson MA, Bresee JS; Global Seasonal Influenza-associated Mortality Collaborator Network. Estimates of global seasonal influenza-associated respiratory mortality: a modelling study. Lancet 2018;391(10127):1285-300. DOI PubMed

18. Boivin G, De Serres G, Hamelin ME, Côté S, Argouin M, Tremblay G, Maranda-Aubut R, Sauvageau C, Ouakki M, Boulianne N, Couture C. An outbreak of severe respiratory tract infection due to human metapneumovirus in a long-term care facility. Clin Infect Dis 2007;44(9):1152-8. DOI PubMed

19. Liao RS, Appelgate DM, Pelz RK. An outbreak of severe respiratory tract infection due to human metapneumovirus in a long-term care facility for the elderly in Oregon. J Clin Virol 2012;53(2):171-3. DOI PubMed

20. Centers for Disease Control and Prevention. Interim guidance for influenza outbreak management in long-term care and post-acute care facilities. Atlanta (GA): CDC: 2019 (accédé 2019-10-25). https://www.cdc.gov/flu/professionals/ infectioncontrol//tc-facility-guidance.htm

21. AHS Outbreak Management Working Group. Guidelines for outbreak prevention, control and management in acute care and facility living sites: includes Influenza and Gastrointestinal Illness. Edmonton (AB): Alberta Health Services; 2019 (accédé 2019-10-25). https://www. albertahealthservices.ca/assets/healthinfo/hi-dis-flu-prov-hlsl. pdf

22. Alberta Health Services. IPC diseases and conditions table: recommendations for management of patients - acute care. Edmonton (AB): AHS; 2019 (accédé 2019-10-25). https:// www.albertahealthservices.ca/assets/healthinfo/ipc/hi-ipcresource-manual-main-document.pdf 